\title{
Richter's Syndrome
}

\author{
Maj K Pathania* \\ MJAFI 2009; 65 : 375-377
}

Key Words : Richter's syndrome; Large cell lymphoma; Chronic lymphocytic leukemia

\section{Introduction}

$\mathrm{R}$ ichter's syndrome $(\mathrm{RS})$ is transformation of chronic lymphocytic leukemia (CLL) to a high grade lymphoma which occurs in up to $10 \%$ of patients with CLL. Mostly, the transformation is into large cell lymphoma but occasionally conversion to prolymphocytic leukemia, small non-cleaved lymphoma, lymphoblastic lymphoma, Hodgkin's disease, hairy cell leukemia and acute leukemia has been noted. This condition was described by Richter in 1928 [1]. However, it came to be known as Richter's syndrome in 1964. It is characterized by worsening of systemic symptoms, rapid tumor growth and/or extra nodal involvement. This is essentially a disease of older age group, with a median survival with conventional chemotherapy of less than six months. Improvement in the outcome of patients with Richter's syndrome may be aided by a more comprehensive understanding of the pathogenesis of Richter's syndrome. Therapy could then be targeted against specific abnormalities.

We report a case of a male patient suffering from CLL who later presented with worsening of symptoms and was diagnosed to have developed large cell lymphoma.

\section{Case Report}

A 63 years old male patient presented with history of fatigue, loss of appetite and weakness. On examination, he had cervical and axillary lymphadenopathy with hepatosplenomegaly. Lymph nodes were rubbery in consistency and largest node measured $4 \times 4 \mathrm{~cm}$. Liver span was $18 \mathrm{~cm}$ in right midclavicular line and it was firm, smooth and nontender. Spleen was just palpable.

Laboratory investigations revealed normocytic, normochromic anaemia with evidence of haemolysis in form of fragmented cells and microspherocytes. His haemoglobin was $8 \mathrm{gm} / \mathrm{dl}$, total leucocyte count $11,200 / \mathrm{mm}^{3}$ and platelet count $1,20,000 / \mathrm{mm}^{3}$. Fine needle aspiration cytology (FNAC) of cervical lymph nodes revealed a monomorphic lymphoid population, composed of small lymphocytes with clumped chromatin, round nucleus and occasional small nucleolus (Fig. 1). Occasional mitosis was present but no necrosis was seen. Provisional diagnosis of chronic lymphocytic leukemia/ small lymphocytic lymphoma (CLL/SLL) was given (Rai stage III). Patient was referred to an oncology centre where lymph node biopsy was carried out and diagnosis was confirmed by histopathology and immunohistochemistry (CD 5 positive, CD 23 positive) (Fig. 3). Patient was put on chemotherapy with chlorambucil.

After about six months, patient again reported with worsening of symptoms in form of wasting, large bilateral cervical and axillary nodes. Largest node in right cervical area measured $5 \mathrm{~cm}$ in diameter. Going through his old treatment record and clinical history, it was learnt that he had discontinued his chemotherapy on his own after three cycles, without consulting the treating oncologist. Ultrasonography revealed hepato-splenomegaly as well as bilateral enlarged matted lymph nodes of cervical and axillary region. FNAC from cervical nodes showed sheets of large lymphoid cells with vesicular nuclei and prominent nucleoli (Fig. 2). Mitotic and atypical mitotic activity was seen along with areas of necrosis. Axillary nodes also showed similar cytological picture. Considering these features, a diagnosis of diffuse large cell lymphoma was offered. Patient was again referred to oncology centre for confirmation by biopsy and further management. Histopathology showed a large cell lymphoma composed of large, singly placed cells in sheets with abundant cytoplasm, vesicular nuclei and prominent eosinophilic nucleoli (Fig. 4).

This was a case of partially treated CLL/SLL which metamorphosised into diffuse large cell lymphoma, hence qualifying as a case of Richter Syndrome.

\section{Discussion}

Richter's Syndrome is the most serious complication of CLL/SLL [2]. The usual presentation is sudden clinical deterioration, asymmetric adenomegaly, hepatosplenomegaly, fever, weight loss and night sweats (B symptoms), increased lactate dehydrogenase (LDH) and monoclonal gammopathy. The most common sites where

*Graded Specialist (Pathology), MH, Trivandrum, Kerala-695006.

Received : 07.07.08; Accepted : 11.05.09_E-mail:aceofspades1018@rediffmail.com 


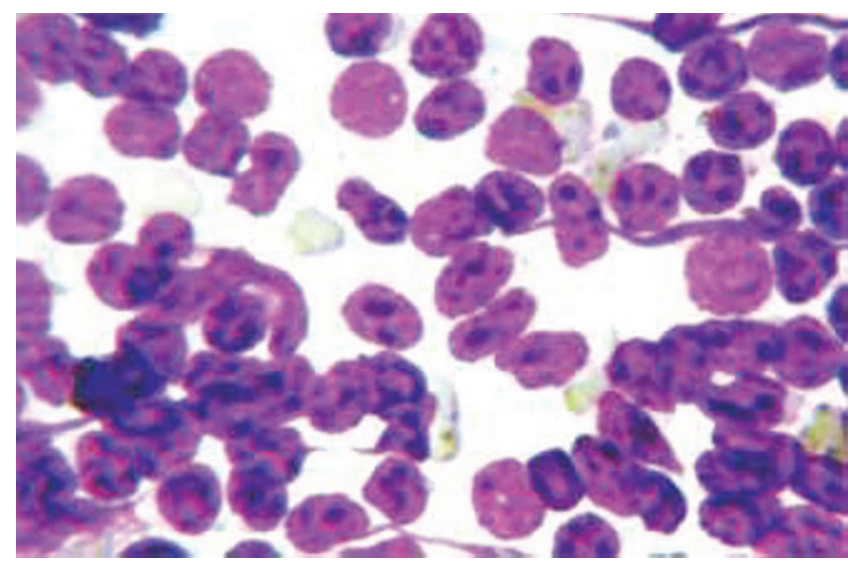

Fig. 1 : Microphotograph showing small lymphoid cells of CLL with clumped chromatin and inconspicuous nucleolus (LG stain, $40 \mathrm{X}$ )

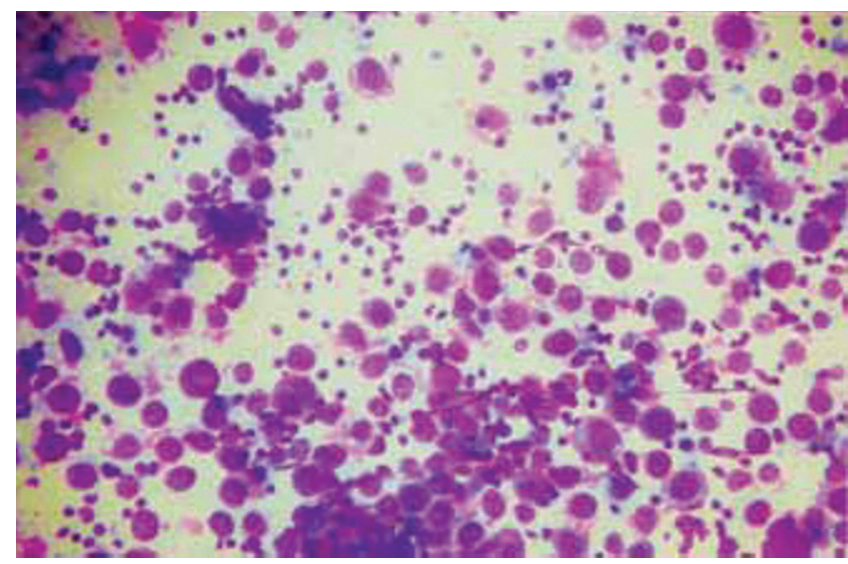

Fig. 2: Microphotograph showing transformed large lymphoid cells with cytoplasm, vesicular nuclei and prominent nucleolus (LG stain, $10 \mathrm{X}$ )

the transformation occurs are the bone marrow or lymph nodes. Although extra-nodal involvement is possible, isolated topography is extremely uncommon [3]. Extranodal sites include the gastrointestinal tract, lung, pleura, oropharynx, bone, skin and central nervous system.

There are no established risk factors for RS, however, younger age, peripheral blood involvement, diffuse involvement of the bone marrow, initial haemoglobin less than $12 \mathrm{~g} / \mathrm{dl}$, advanced Rai stage, increasing LDH levels and high beta- 2 microglobulin levels are considered possible risk factors.

There are no specific laboratory findings for RS. There may be elevation of LDH levels, which can be a marker of tumor growth. $44 \%$ of patients in one study presented with paraproteinemia [4]. Lytic bone lesions or hypercalcemia may be present, probably due to increased bone resorption. In some patients, the presence of circulating immunoblasts in the peripheral blood is the first indication of RS. The histologic diagnosis is made by the presence of large, round or slightly irregular cells with moderate amounts of cytoplasm, vesicular nuclei

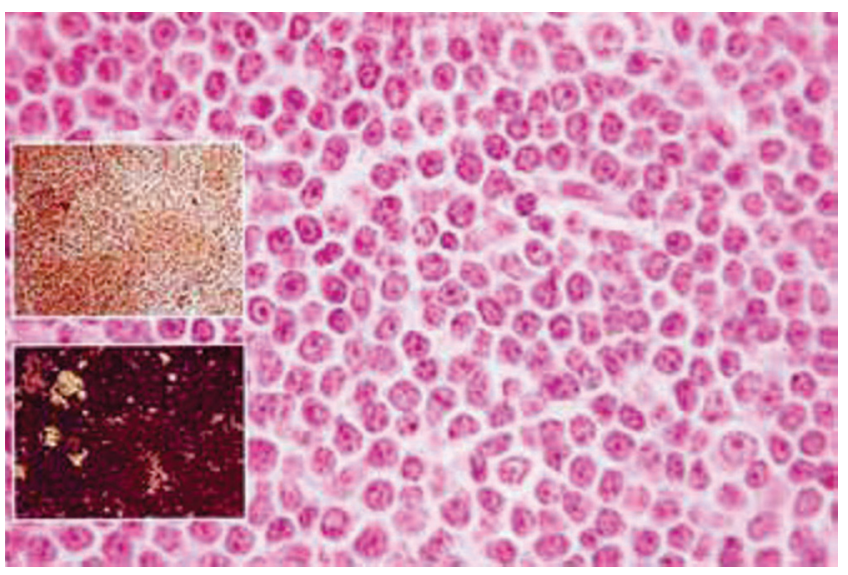

Fig. 3 : Microphotograph showing small lymphoid cells lying singly with clumped chromatin and inconspicuous nucleolus (H\&E, $40 \mathrm{X}$ ). Inset pictures shows CD 23 positivity (top and CD 5 positivity (below)

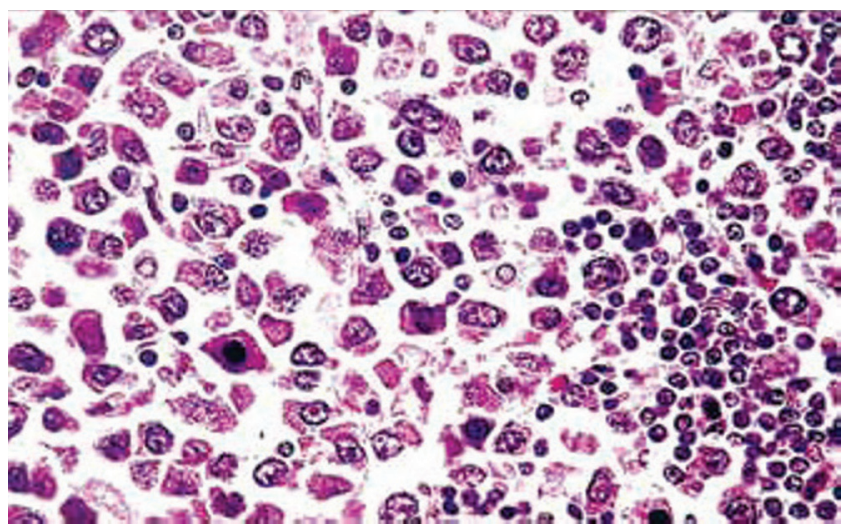

Fig. 3 : Microphotograph showing transformed large lymphoid irregular cells with cytoplasm, vesicular nuclei and prominent nucleolus (H\&E, $10 \mathrm{X}$ )

and prominent nucleoli that are distinct from those of CLL/ SLL cells. The specimen's architecture can be completely effaced by the large cell lymphoma or the latter can coexist with CLL/SLL cells. A variant of RS has been described when patients with CLL/ SLL develop Reed-Sternberg like cells, and this is termed Hodgkin variant of RS.

However, despite the well-known occurrence of this phenomenon, the precise clonal relationship between the two neoplastic cell populations is still unclear. It has been suggested, based on immuno-histologic, immunoglobulin isotype, anti-idiotype, Ig gene rearrangement and cytogenetic studies that the large cell lymphoma develops as a clonal evolution from CLL/ SLL [5]. Clonal evolution may be triggered by a viral infection like Ebstein Barr virus, especially in immunocompromised patients. Other studies suggest that the two neoplasms are distinct, unrelated clonal proliferations [6]. Unfortunately, a definitive conclusion cannot be drawn from these conflicting reports [7]. Oncogenesis of RS may occur through trisomy $12,11 \mathrm{q}$ structural 
abnormalities, microsatellite instability and tumor suppressor gene mutations.

The treatment for RS ranges from chemotherapy to transplantation. Currently, RS is considered to be resistant to conventional combined chemotherapeutic agents. Commonly used protocol is combined chemotherapy with cis-platinum, fludarabine and cytosine arabinoside, or substitution of cyclophosphamide instead of cis-platinum. HyperCVXD (fractionated cyclophosphamide, vincristine, liposomal daunorubicin and dexamethasone) is also being used increasingly with more favorable results. Postchemotherapy allogeneic stem cell transplantation has been shown to achieve higher remission rates. The management of CNS RS includes systemic and intrathecal chemotherapy and radiotherapy. The role of radiotherapy is limited to control pain and symptoms associate with bulky lymphadenopathy or extra nodal disease. Patients with Hodgkin variant of RS are more resistant to therapy than those with classic RS.
Conflicts of Interest

None identified

\section{References}

1. Richter MN. Generalized reticular cell sarcoma of lymph nodes associated with lymphatic leukemia. Am J Pathol 1928; 6 : 285-99.

2. O’Neill BP, Habermann TM, Banks PM, et al. Primary central nervous system lymphoma as a variant of Richter's syndrome two patients with chronic lymphocytic leukemia. Cancer 1989; 15: 1296-1300.

3. Robertson LE, Pugh W, O'Brien S, et al. Richter's syndrome: a report of 39 patients. J Clin Oncol 1993; 11:1985-9.

4. Tsimberidou AM, Keating MJ. Richter syndrome: biology, incidence, and strategies. Cancer 2005; 103: 216-28.

5. Nowell P, Finan J, Glover D, Guerry D. Cytogenetic evidence for the clonal nature of Richter's syndrome. Blood 1981; 58: 183-6.

6. McDonnell JM, Beschorner WE, Staal SP, et al. Richter's syndrome with two different B-cell clones. Cancer 1986; 58:2031-7.

7. Foon KA, Thiruvengadam R, Saven A, et al. Genetic relatedness of lymphoid malignancies. Transformation of chronic lymphocytic leukemia as a model. Ann Intern Med 1993; 119:63-73.

\section{Answers to Picture Quiz}

Answer 1 : "Setting-Sun or Sun-Setting" sign. The pictures are of a nine-month-old baby with hydrocephalus with a head circumference of 63 centimeters at presentation.

Answer 2 : The eye sign is so named because of the typical appearance of the cornea to a setting sun due to clearly visible sclera above the upper margin of cornea with inferior portion hidden behind the lower eyelid margin. However, at presentation this infant's corneae were completely invisible and were hidden behind the lower eyelid margin due to extreme downward displacement of the eyes suggesting "set-sun" sign rather than " sun-setting" sign (Fig.1). Neuroimaging revealed gross hydrocephalus with aqueductal stenosis as the likely etiology. After adequate supportive and symptomatic therapy, a ventriculo-peritoneal shunt was placed. Rapid "shrinking" of the head followed this and cornea re-appeared gradually above the lower lid margins mimicking "rising-sun" (Fig.2). The difference between the two images is that, when Fig. 1 is seen in isolation, particularly only close-up of the eyes, it would be difficult to say that it is an eye-sign of hydrocephalus as cornea is not seen at all! Also, if the history of surgery is not given, it is not possible to differentiate between "sun-setting" or "rising- sun" variant by looking at only Fig. 2.

Answer 3 : This sign appears as a result of downward displacement of the eyes due to impingement of dilated suprapineal recess on the tectum resulting in upward gaze palsy. As the eyeballs are displaced downwards, a clear margin of sclera becomes visible above the upper border of cornea and the inferior border is hidden beneath the lower lid margin. What we would like to bring out through these pictures is an interesting phenomenon observed by us, where in, it may be appropriate to call this eye-sign as already "set-sun" with "rising-sun" appearance after relief of raised intracranial pressure. We attribute this to extreme degree of downward displacement of the eyeballs due to prolonged, unrelieved obstruction to CSF drainage. With shunt placement, the pressure on the tectum was relieved to a large extent resulting in a sequential "set-sun" - "sun-rise" sign. Based on available explanation for this sign, we hypothesize that if the obstruction is not relieved in hydrocephalus and the child survives, the sequential eye signs could be "setting-sun" followed by "set-sun" subsequently leading on to "rising-sun" appearance, after obstruction is relieved. We further clarify here that the feature described by us is not a new sign, it is an extreme and rare variant of already described eye sign in children with gross hydrocephalus.

Fig. 1 : Infant at presentation showing massive hydrocephalus with cornea completely hidden under the lower eye lid- Set-Sun sign.

Fig. 2: The cornea became visible with typical "Rising-Sun" sign after relief of obstruction through the placement of a ventriculo-peritoneal shunt. 